\title{
THE EFFECT OF NONLINEAR SYNCHROTRON MOTION ON THE SOLEIL ENERGY ACCEPTANCE *
}

\author{
$\underline{A}^{\text {N Nadji }^{\dagger}}{ }^{\dagger}$ J.- L. Laclare, M.- P. Level, A. Mosnier, P. Nghiem, \\ Projet SOLEIL, DRIF CNRS, Av. de la Terrasse, Bât. 5, 91198 Gif sur Yvette, France \\ G. Flynn, LURE, Centre Universitaire Paris-Sud, Orsay, France
}

\section{Abstract}

The lengthening of the trajectory is determined by the momentum compaction factor $\alpha$ and the betatron oscillation contribution term $\frac{\Delta C}{C_{0}}$. When the first order term of the momentum compaction factor $\alpha_{1}$ is small, the second order term $\alpha_{2}$ and $\frac{\Delta C}{C_{0}}$ have to be included in the longitudinal equations of motion. In this case, the RF bucket form changes and the energy acceptance can be significantly reduced. This leads to a decrease in Touschek beam lifetime. In this paper we show that the value of $\alpha_{2}$ in the standard low emittance lattice of SOLEIL is large and can reduce the energy acceptance of the machine. We discuss the magnitude of this reduction and the difficulties encountered in minimizing the value of $\alpha_{2}$ in the case of a low emittance lattice. We also show that the lengthening of the trajectory induced by horizontal and vertical betatron oscillations vanishes when the chromaticities are corrected to zero.

\section{INTRODUCTION}

In a storage ring, the Touschek relevant energy acceptance may be determined by the RF bucket momentum height, by the physical aperture or by the dynamic aperture associated with off momentum particles if the induced amplitude after a Touschek scattering event exceeds one of these two transverse limits. In the case of the SOLEIL project, a great effort has been made to increase the dynamic aperture for off-momentum particles leading to a transverse energy acceptance of up to $\pm 6 \%$ [1]. The Touschek beam lifetime calculations take into account the higher order effects in energy which become important for such large values [2]. The use of superconducting cavities with a peak voltage fixed at 3.8 MV insures a longitudinal energy acceptance larger than $\pm 6 \%$. Nevertheless, this value was determined using the first order momentum compaction factor, $\alpha_{1}$, thus assuming that the other momentum factor terms are negligeable. In our case, the second order term of the momentum compaction factor $\alpha_{2}$ is significant and must be considered in the equations of longitudinal motion. The expected problem is that the synchrotron motion being nonlinear, the buckets become asymmetric which can reduce the RF energy acceptance and therefore the Touschek beam lifetime.

\footnotetext{
${ }^{*}$ Work supported by CNRS-CEA-MENESR
}

† Email: Nadji@ lure.u-psud.fr

\section{NONLINEAR LONGITUDINAL MOTION}

The momentum compaction factor is defined as the relative change in orbit path length, $\frac{\Delta l}{l_{0}}$, with relative particle energy, $\delta=\frac{\Delta p}{p_{0}}$ :

$$
\alpha=\frac{d\left(\Delta l / l_{0}\right)}{d \delta}
$$

where $p_{0}$ is the momentum of the reference particle and $l_{0}$ the length of the reference orbit.

The expression for particle position contains betatron oscillation, closed orbit distortion and off momentum orbit terms :

$$
x=x_{\beta}+x_{c o}+\eta_{1} \delta+\eta_{2} \delta^{2}+\ldots
$$

$\eta_{1}$ and $\eta_{2}$ are respectively the first and the second order terms of the dispersion function. Assuming that $x_{c o} \approx 0$, the resulting variation in orbit length with energy (up to the second order) and betatron amplitudes, can be written in the following way:

$$
\frac{\Delta l}{l_{0}}=\frac{\Delta C}{C_{0}}+\alpha_{1} \delta+\alpha_{2} \delta^{2}
$$

where

$$
\begin{gathered}
\frac{\Delta C}{C_{0}}=\frac{1}{l_{0}} \oint\left(\frac{x_{\beta}}{\rho}+\frac{x_{\beta}^{\prime 2}}{2}\right) d s \\
\alpha_{1}=\frac{1}{l_{0}} \oint \frac{\eta_{1}}{\rho} d s \quad \alpha_{2}=\frac{1}{l_{0}} \oint\left(\frac{\eta_{2}}{\rho}+\frac{\eta_{1}^{\prime 2}}{2}\right) d s
\end{gathered}
$$

$\frac{\Delta C}{C_{0}}$ is the term representing lengthening effects due to betatron oscillations, $\alpha_{1}$ and $\alpha_{2}$ are respectively the first and the second order terms of the momentum compaction factor.

The longitudinal motion equations including these terms can then be written as :

$$
\begin{aligned}
\frac{d \varphi}{d t} & =-\omega_{r f}\left[\alpha_{1} \delta+\alpha_{2} \delta^{2}+\frac{\Delta C}{C_{0}}\right] \\
\frac{d \delta}{d t} & =\frac{e V_{r f}}{E T}\left[\sin \varphi-\sin \varphi_{s}\right]
\end{aligned}
$$

they can be derived from the following Hamiltonian :

$$
\begin{gathered}
H(\delta, \varphi)=\frac{\Delta C}{C_{0}} \delta+\alpha_{1} \frac{\delta^{2}}{2}+\alpha_{2} \frac{\delta^{3}}{3}+ \\
\frac{e V_{r f}}{E T}\left(\cos \varphi-\cos \varphi_{s}+\left(\varphi-\varphi_{s}\right) \sin \varphi_{s}\right)
\end{gathered}
$$


where $\omega_{r f}$ is the angular frequency of the RF cavity, $V_{r f}$ is the RF peak voltage, $E$ is the particle energy and $T$ the revolution period, $\varphi_{s}$ and $\varphi$ are respectively the phases of the reference and the test particles.

In the case where $\alpha_{2} \neq 0$ and $\frac{\Delta C}{C_{0}} \neq 0$ we obtain two stable fixed points and two unstable fixed points which correspond to the existence of a second zone of stability in addition to the well-known stable linear RF-bucket. According to the values of $\alpha_{2}$ and $\frac{\Delta C}{C_{0}}$, the synchrotron diagram presents different aspects, particularly the separatrices are no longer symmetric in $+\delta$ and $-\delta$ [3],[4].

\section{THE CASE OF THE SOLEIL OPTICS}

The small beam emittance is obtained by increasing the amount of horizontal focusing. The dispersion in the bending magnets is then very small and consequently we find naturally small values of $\alpha_{1}$. At its nominal energy, $2.5 \mathrm{GeV}$, the lattice has a horizontal emittance of $3 \mathrm{~nm}$.rad. In this case $\alpha_{1}$ is about $4.722 \times 10^{-4}$ and using the values of the sextupoles corresponding to the optimized dynamic aperture the value of the $\alpha_{2}$ term is about $4.513 \times 10^{-3}$.

Figure 1 shows the longitudinal phase space diagram corresponding to these two values but assuming that $\frac{\Delta C}{C_{0}}=0$.

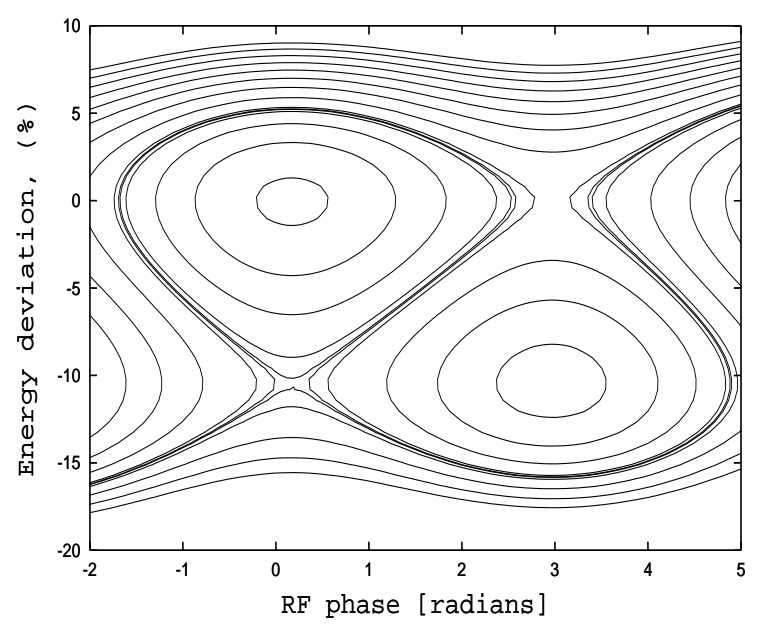

Figure 1: Longitudinal phase space.

We can see that the effect of $\alpha_{2}$ is important. The RFbucket, centered around $\delta=0$., is asymmetric in energy, the upper limit is at $+5.2 \%\left(\left|\frac{\alpha_{1}}{2 \alpha_{2}}\right|\right)$ and the lower limit is at $-10.5 \%\left(-\left|\frac{\alpha_{1}}{\alpha_{2}}\right|\right)$. The impact of this asymmetry can be understood by inspecting figure 2 which shows the dynamic and physical apertures calculated as a function of $\delta$. We recall that in the linear case, the RF bucket height is about $\pm 6 \%$. In figure 2 , one can see that a particle with an energy deviation of $+6 \%$ is still inside the lowest transverse aperture (the vacuum chamber aperture) when its energy deviation changes to $-6 \%$. Unfortunately, the low value of $\alpha_{1}$ renders significant the contribution of the term $\alpha_{2}$ and the synchrotron motion becomes in fact nonlinear. A particle with a positive energy deviation of $+5.2 \%$ for example can be lost because its separatrix can bring it to a negative energy deviation as high as $-10.5 \%$.

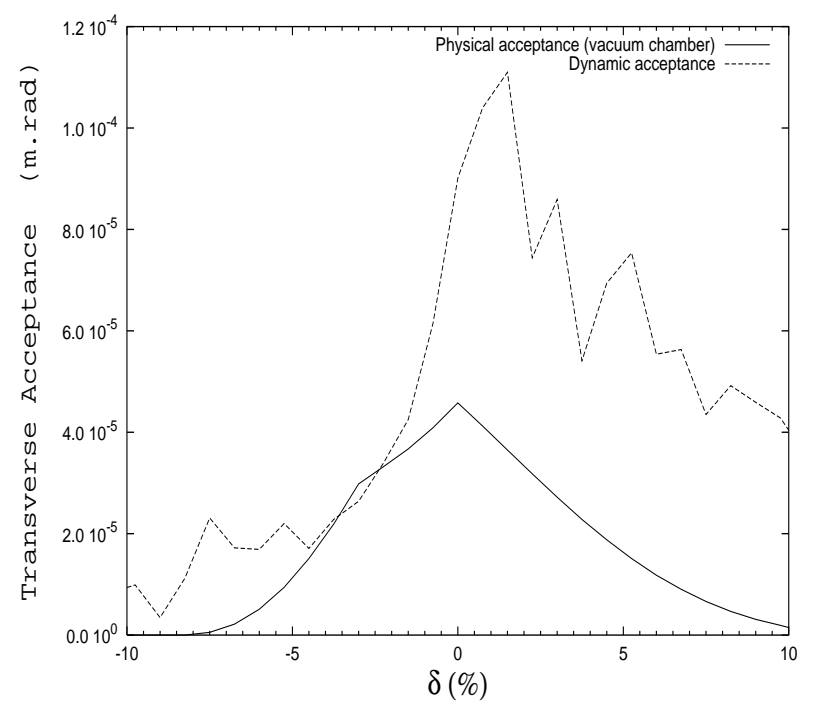

Figure 2: Horizontal dynamic and physical acceptances as a function of $\delta$.

The energy acceptance/Touschek lifetime module of the BETA code [5], described in detail in [2] has been modified to take into account the non-linear synchrotron motion. Instead of assuming, as we did in [2] that a particle having been Touschek scattered to $\delta$ will oscillate between $\delta$ and $-\delta$ during one synchrotron period, we now calculate the mapping, by integration of the longitudinal equations (6), from $\delta$ to the maximum opposing energy $\delta_{\max }(\delta)$ which occurs one half synchrotron period later. Expression (3) of [2] then becomes :

$$
A(\delta)=\min _{\delta^{\prime} \in\left[\delta, \delta_{\max }(\delta)\right]}\left\{\min \left[A_{\text {phys }}(\delta), A_{\text {dyn }}(\delta)\right]\right\} .
$$

We also take into account the asymmetric longitudinal acceptance which is determined during the process of calculating $\delta_{\max }(\delta)$.

Figure 3 presents the results for the energy acceptance along one period of the ring. The corresponding value of the Touschek beam lifetime is about 26 hours instead of 40.5 hours in the linear case. This calculation is performed for multibunch operation using the following parameters : the bunch current is $1.26 \mathrm{~mA}$, the horizontal-vertical coupling is 0.01 and the natural bunch length is $11.6 \mathrm{ps}$.

To reduce synchrotron motion nonlinearity and thus recuperate some extra beam lifetime, we must reduce the value of $\alpha_{2}$. The expression giving this term shows that, due to the $\eta_{1}^{\prime 2}$ contribution, $\alpha_{2}$ is always positive in a linear machine. $\eta_{2}$ can be made positive or negative with sextupoles and can compensate the term $\eta_{1}^{\prime 2}$. The adjustment of $\alpha_{2}$ by the sextupoles can be made as follows :

$$
\Delta \alpha_{2}=-\frac{\Delta S \eta_{1}^{3}}{l_{0}}
$$




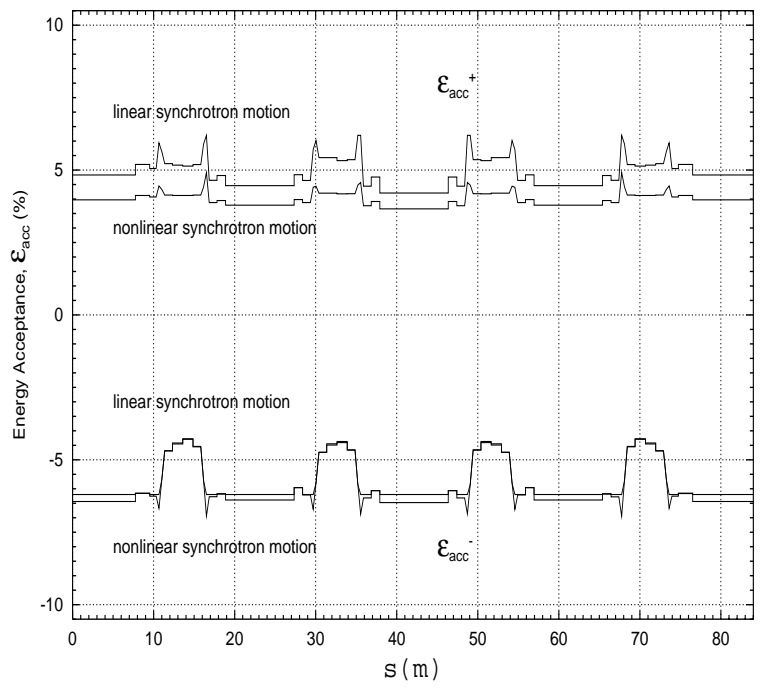

Figure 3: Energy acceptances.

where $\Delta S\left(\mathrm{~m}^{-2}\right)$ is the change in the sextupole strength, $\eta_{1}(m)$ is the horizontal dispersion at the sextupole and $l_{0}(m)$ is the orbit length. Even if we take the sextupole (S4) [1], where the dispersion is maximum $(0.27 \mathrm{~m})$, its efficiency in reducing $\alpha_{2}$ is small. As an example, increasing the strength of the 16 type S4 sextupoles by a factor 2 would reduce $\alpha_{2}$ by only a factor 2 , which is not nearly enough to restore the linearity of the longitudinal motion. The objective is to reduce the term $\alpha_{2}$ while compensating the two transverse chromaticities and obtaining a very good dynamic aperture. This seems to be extremely difficult in the case of a very low emittance lattice for which the dispersion is low and the circumference is large. Up to now, we have not performed an exhaustive study in this direction.

\section{EFFECT OF THE TERM $\frac{\Delta C}{C_{0}}$}

When $\frac{\Delta C}{C_{0}} \neq 0$, the longitudinal equations (6) have the following stable fixed points :

$$
\delta_{1,2}=-\frac{\alpha_{1}}{2 \alpha_{2}}\left(1 \pm \sqrt{1-\frac{4 \alpha_{2}}{\alpha_{1}^{2}} \frac{\Delta C}{C_{0}}}\right)
$$

whose locations depend on the value of the betatron amplitude and the closed orbit. In order for the fixed points to be real we must require :

$$
\frac{\Delta C}{C_{0}}<\frac{\alpha_{1}^{2}}{4 \alpha_{2}} ; \quad \frac{\Delta C}{C_{0}}<1.235 \times 10^{-5}
$$

First, we can determine the maximum RF frequency variation, by taking into account the well-known relation $\frac{\Delta C}{C_{0}}=-\frac{\Delta f_{r f}}{f_{r f}}$ and using expression (11), we can see that there is a limit for $\frac{\Delta f_{r f}}{f_{r f}}<0$. This limit is $\left|\Delta f_{r f}\right|<4.3 \mathrm{kHz}$.
Secondly, in a linear machine (i.e. sextupoles and higher multipoles off), the betatron motion can be represented by $x_{\beta}(s)=\sqrt{a_{0} \beta_{x}(s)} \cos \varphi_{x}(s)$. Introducing this expression and its derivative into formula (4), one can easily obtain an average value for the term $\frac{\Delta C}{C_{0}}$ which is in this case always positive :

$$
\frac{\Delta C}{C_{0}}=\frac{x_{\beta}^{2}}{4 \beta_{x}}<\gamma_{x}>
$$

During the injection of the beam or after a Touschek collision for example, the particle can undergo large amplitude betatron oscillations, the combination of equations (11) and (12) gives an upper limit to $x_{\beta}$.

This could have been a problem without the fortunate effect of chromatic sextupoles. In fact, using the BETA code, we have calculated the variation of $\frac{\Delta C}{C_{0}}$ as a function of the amplitude $x_{\beta}$ for the case where the two transverse chromaticities are corrected to zero. In contrast to the linear case, the average value (over several turns) of $\frac{\Delta C}{C_{0}}$ is very near to zero. The analytical developments taking into account the presence of sextupoles corroborate perfectly the simulations performed with the BETA code which show that the term $\frac{\Delta C}{C_{0}}$ is on average zero when the transverse chromaticities of a storage ring are corrected to zero. In fact the analytical result gives in this case the following expression :

$$
\frac{\Delta C}{C_{0}}=-\pi \frac{x_{\beta}^{2}}{\beta}\left[\xi_{\text {sextupoles }}+\xi_{\text {quadrupoles }}\right]
$$

where $\xi_{\text {quadrupoles }}$ is the natural chromaticity of the ring and $\xi_{\text {sextupoles }}$ is that due to the chromatic sextupoles. As is well known a zero chromaticity corresponds to $\xi_{\text {quadrupoles }}+\xi_{\text {sextupoles }}=0$.

\section{CONCLUSION}

Because of the very low emittance, the effect of $\alpha_{2}$ is significant in the SOLEIL lattice. It reduces the Touschek lifetime by a factor 1.5. Although the correction of $\alpha_{2}$ term seems to be difficult in such lattice, further reflexions on how to reduce its effect are needed.

\section{REFERENCES}

[1] P. Nghiem, et. al., "Optics for SOLEIL at $2.5 \mathrm{GeV}$ ", Proceedings 6th European Particle Accelerator Conference, Stockholm, (1998).

[2] A. Nadji et. al., " Energy acceptance and Touschek lifetime calculations for the SOLEIL project", Proceedings 6th European Particle Accelerator Conference Stockholm, (1998).

[3] A. Nadji, et. al., "Quasi-isochronous experiments with the Super-ACO storage ring ", N.I.M. A378 (1996) 376.

[4] J. P Bardin, "Thesis, 1973".

[5] J. Payet, "BETA-LNS code". 